\title{
Supersymmetric solution of gauge hierarchy problem
}

\author{
ROMESH K KAUL \\ Centre for Theoretical Studies, Indian Institute of Science, \\ Bangalore 560 012, India \\ MS received 11 March 1982
}

\begin{abstract}
Some recent developments with respect to the resolution of the gauge hierarchy problem in grand unified theories by supersymmetry are presented. A general argument is developed to show how global supersymmetry maintains the stability of the different mass-scales under perturbative effects.
\end{abstract}

Keywords. Gauge hierarchy problem; supersymmetry.

It has been known for sometime now that the elementary scalars may make a field theory 'unnatural' (Susskind 1979; Wilson as quoted therein). The concept of naturalness may be understood as the requirement that smallness of a parameter in a theory should not be a mere accident but should rather follow from a weakly broken symmetry so that its smallness is protected against small perturbations ('t Hooft 1979). In the standard grand unification models, this is also related to an important problem, the gauge hierarchy problem (Gildner and Weinberg 1976; Gildner 1976 and 1980). In the ordinary scenario of grand unification, where there are more than one distinct mass scales introduced through the vacuum expectation values of a suitably chosen set of scalar fields, the mass-scales tend to merge into each other under perturbative effects. This spoils the distinction between the light and the heavy scales. The origin of this problem resides in the presence of quadratically divergent mass corrections and also logarithmically divergent mass corrections with large coefficients to the light scalar masses.

The resolution of the problem may be conceived of in two ways:

(i) No elementary scalar fields: the scalar fields are thought to be fermion-antifermion composites. This is the well-known technicolour solution which we shall not discuss here (for a review see Kaul 1981b).

(ii) Scalar fields are elementary but naturalness violating effects are arranged to be absent. In this context, it is important to notice that the corrections due to the graphs with a fermionic loop and a bosonic loop have opposite signs. Hence, we may be able to allow them to conspire against each other so that unwanted effects are cancelled out. However, this has to happen with extreme precision. Say, for a Higgs mass $\approx 100 \mathrm{GeV}$ and a physical cut-off $\approx 10^{15} \mathrm{GeV}$ (grand unification scale), this cancellation would have to be as accurate as one in $10^{-4} \times 10^{30}=10^{26}$ ! Besides, this will have to be valid at loop-to-loop level. This tight-rope balance suggests imposition of a symmetry. The symmetry that would connect the fermionic and bosonic effects is supersymmetry. This is precisely the motivation as to why super- 
symmetry may provide a solution, and, indeed, it does, as we shall see in the following.

Absence of quadratic divergent corrections to the masses in Wess-Zumino scalar multiplet model was noticed long ago (Wess and Zumino 1974a, b; Illiopoulos and Zumino 1974). This is also true in the supersymmetric extension of QED (Wess and Zumino 1974c; Ferrara and Piquet 1975). The reason for this can be easily appreciated if we realize that the fermion self-energy are only logarithmically divergent and hence supersymmetry would ensure absence of quadratic divergences also in the self-energies of the bosons which are the super-partners of these fermions. The situation is not so clear with models with spontaneously broken gauge symmetry, because tadpole graphs here would be responsible for giving quadratic divergent corrections to the fermions even in 't Hooft-Feynman gauge. However, explicit calculations show that even in ABJ anomaly-free spontaneously broken gauge theories such divergences are indeed absent (Kaul and Majumdar 1981a, b). The necessary and sufficient condition for the absence of these divergences is absence of the ABJ anomalies (Kaul and Majumdar 1981a, b; Fischler et al 1981). This also ensures absence of quadratic divergences at higher loop levels if these are absent at one loop level through Adler-Bardeen theorem which states that the anomalies are generated only at one loop level.

Separate cancellation of the quadratic and the large logarithmic divergent corrections to smaller masses can also be shown to obtain in anomaly-free models with two distinct mass scales. This has been explicitly demonstrated in an $\mathrm{SU}(2) \times \mathrm{U}(1)$ model spontaneously broken down to no symmetry in our earlier paper (Kaul 1981). This, hence, would allow us to preserve the distinction between the light and the heavy mass scales. In the following we shall present a new, more general argument, for the absence of large corrections for a general gauge group, without resorting to detailed explicit calculations.

Consider an anomaly-free model with gauge group $G$ which is broken at two levels

$$
G \stackrel{M_{H}}{\rightarrow} G_{1} \stackrel{M_{L}}{\longrightarrow} G_{2}
$$

by allowing two sets of scalar fields get vacuum expectation values $F$ and $f$ $(F>f)$, respectively. Let $H_{1}$ and $H_{2}$ be the generic physical (shifted by the v.e.v's). Higgs fields corresponding to these two symmetry breakings, with their tree level masses $M_{1}^{2} \equiv M_{H}^{2} \sim F^{2}$, and $M_{2}^{2} \equiv M_{L}^{2} \sim f^{2}$. The various gauge bosons corresponding to the broken generators at these two levels would pick up masses of the order $F^{2}$ and $f^{2}$ respectively, so would the fermions which are suitably coupled to these scalar fields through Yukawa couplings. The gauge hierarchy is $O\left(M_{1}^{2} / M_{2}^{2}\right)$ $=O\left(F^{2} / f^{2}\right)$ at the tree level.

In terms of the shifted fields $H_{1}, H_{2}$, the Higgs potential at the tree level would read

$$
V\left(H_{1}, H_{2}\right)=\frac{1}{2} M_{1}^{2} H_{1}^{2}+\frac{1}{2} M_{2}^{2} H_{2}^{2}+a H_{1}^{3}+b H_{2}^{3}+c H_{1}^{2} H_{2}+d H_{1} H_{2}^{2}
$$

+ quartic couplings 
where $\left.\quad \begin{array}{rl}M_{1} & =O(F), M_{2}=O(f) \\ a & =O(F), b=O(f) \\ c & =O(f), d=O(F) .\end{array}\right\}$

Here we have not written the $H_{1}, H_{2}$ mixed term, because we assume that it has been absorbed by diagonalization of the $H_{1}, H_{2}$ mass matrix. In addition, there would also be various interactions of the Higgs $H_{1}, H_{2}$ with the gauge bosons and the fermions. We do not need to know the exact form of these interactions for our argument.

Now, after fixing the gauge (say, 't Hooft-Feynman gauge), we may attempt to compute the one-loop corrections to the masses of $H_{1}$ and $H_{2}$. Here we would come across the following three types of graphs of interest:

(i) Tadpole graphs with $H_{1}$ and $H_{2}$ external lines as shown in figure 1 which yield the terms

$$
2 \beta_{1} H_{1}+2 \beta_{2} H_{2},
$$

in the one-loop effective potential where $\beta_{1}$ and $\beta_{2}$, both quadratically divergent, are at the strongest of order $F^{3}$ and $f F^{2}$ respectively, depending upon the strength of the vertex and the mass of the field $\Phi_{i}$ (scalar, vector, fermion, or Fadeev-Popov ghost) going around the loop. In particular for diagrams with $H_{1}, H_{2}$ in the loop,
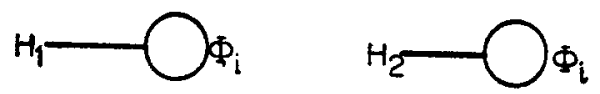

[I]

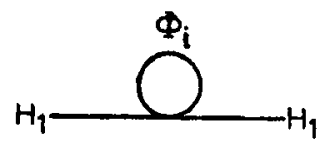

(a)

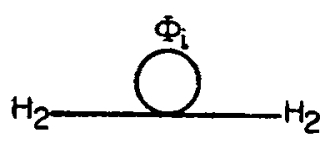

(c)

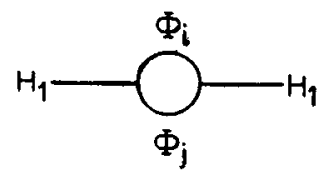

(b)<smiles></smiles>

(d)<smiles></smiles>

(iII)

Figures 1 to 3. Various one-loop graphs relevant to the calculation of corrections to the scalar masses. 
this can be directly seen by putting in the various values of masses and couplings as given in (2).

(ii) $H_{1}-H_{1}$ and $H_{2}-H_{2}$ self-energy graphs as shown in figure 2. The graphs in figures $2 \mathrm{a}$ and $2 \mathrm{~b}$ are quadratically divergent and hence contribute at the strongest $O\left(F^{2}\right)$ terms from those fields $\Phi_{i}$ (scalars, vectors) with masses $O\left(F^{2}\right)$ that may couple to the field in question. Graphs $2 \mathrm{~b}$ and $2 \mathrm{~d}$ have only logarithmic divergences if the internal lines $\Phi_{i}, \Phi_{\text {, }}$ are both scalars or Fadeev-Popov ghosts, some of them would also be $\sim F^{2}$ due to large three-point couplings such as $a$ and $d$ in the potential (1). When $\Phi_{i}, \Phi_{j}$ are both fermions or one vector and other scalar field, we have in addition quadratically divergent parts, which may also contribute $O\left(F^{2}\right)$ to $H_{1}-H_{1}$ and $\mathrm{H}_{2}-\mathrm{H}_{2}$ self-energy.

(iii) Mixed $H_{1}-H_{2}$ two-point function as shown in figure 3. Here we have contributions $\sim F f$ as can be seen from effective potentials (1) and (2) for $\Phi_{i}=H_{1}, \Phi_{j}=H_{2}$ or $\Phi_{i}=\Phi_{j}=H_{1}$ or $H_{2}$.

Now we may shift the fields in the one-loop effective potential to absorb the effect of the tadpole graphs (3) by replacing

$$
\begin{aligned}
& H_{1} \rightarrow H_{1}+2 \beta_{1} / M_{1}^{2}, \\
& H_{2} \rightarrow H_{2}+2 \beta_{2} / M_{2}^{2} .
\end{aligned}
$$

In terms of these shifted fields the quadratic terms in the one-loop effective potential up to the lowest order in coupling can be written as

$$
V_{2}\left(\tilde{H}_{1}, \tilde{H}_{2}\right)=\frac{1}{2} \tilde{M}_{1}^{2} \tilde{H}_{1}^{2}+\frac{1}{2} \tilde{M}_{2}^{2} \tilde{H}_{2}^{2}+\tilde{M}^{2} \tilde{H}_{1} \tilde{H}_{2}
$$

where, taking into account the various contributions from the three types of graphs 1 to 3 listed above

$$
\begin{aligned}
& \tilde{M}_{1}^{2}=F^{2}\left[a+O\left(f^{2} / F^{2}\right)\right], \\
& \tilde{M}_{2}^{2}=F^{2}\left[\beta+O\left(f^{2} / F^{2}\right)\right], \\
& \tilde{M}^{2}=F f\left[\gamma+O\left(f^{2} / F^{2}\right)\right] .
\end{aligned}
$$

Notice our new fields $\tilde{H}_{1}, \tilde{H}_{2}$ have the proper wave-function renormalizations absorbed in them.

In formulae (6) the strongest order entry in $\tilde{M}^{2}$ is only $O(f F)$ unlike in $\tilde{M}_{1}^{2}$ and $\tilde{M}_{2}^{2}$, where it is $O\left(F^{2}\right)$.

Now let us take the limit $f^{2} \rightarrow 0$ by appropriate adjustment of the parameters in the original Higgs-Potential. In this limit, the second symmetry breaking, $G_{1} \stackrel{f}{\rightarrow} G_{2}$, does not happen; $G_{1}$ symmetry is restored provided the radiative corrections do not break this symmetry. In this case the masses of all the particles corresponding to the second symmetry breaking, in this limit, would become zero. In order this be consistent with one-loop effective potential, we must have $\beta=0$ in (6). If so 
then for the case $f^{2} \neq 0$, when $G_{1}$ is broken down to $G_{2}$ spontaneously, $\beta=0$ is still true and $\tilde{M}_{1}^{2}=O\left(F^{2}\right), \tilde{M}_{2}^{2}=O\left(f^{2}\right)$ and $\tilde{M}^{2}=O(f F)$. Hence we can maintain the distinction between light and heavy particles even after quantum effects have been taken into account, i.e., the gauge hierarchy can be maintained at higher loop level also once properly chosen at the tree level.

However, this argument is valid only for supersymmetric theories. We know that in a field theory where the Higgs potential at the tree level has a vanishing curvature at the origin and a nonzero four-point scalar self-interaction and also gauge interaction of the scalars, the radiative effects induce a symmetry breaking (Coleman and Weinberg 1973). However, this Coleman-Weinberg mechanism of symmetry breaking by radiative correction does not obtain in an $A B J$ anomaly-free globally supersymmetric theory. This can be seen by a straightforward calculation of the oneloop radiative corrections to the potential in Landau gauge in the manner of Coleman and Weinberg. Each supermultiplet contributes zero correction to every function with $2 n$ zero momentum external scalar lines. This result, needless to say, would be valid at all loop levels.

To conclude, in an anomaly-free supersymmetric theory radiative corrections do not break the gauge symmetry and hence our argument given above holds for this case; the mass scales are stable under perturbative effects. On the other hand in the case of non-supersymmetric theories, the radiative corrections break the symmetry and hence stability of mass scales does not obtain.

To understand this result from 't Hooft's naturalness point of view, we realize that $f^{2} \rightarrow 0$ does enhance the symmetry (we have $G_{1}$ symmetry) in the supersymmetric case whereas in the non-supersymmetric case it does not, because the radiative corrections break this symmetry $a$ la Coleman and Weinberg, even if at the tree level the symmetry did exist. Hence in the supersymmetric case only $f^{2}$ can be allowed to be small.

In the last one year a large number of related papers presenting similar ideas have appeared. Some of these are, Veltman (1980), Dimopoulos et al (1981), Sakai (1981), Dimopoulous and Georgi (1981), Witten (1981), Fischler et al (1981) and Dine et al (1981).

\section{Acknowledgement}

Discussions with P Majumdar, J Pasupathy, Ashok Raina, R Rajaraman, P Roy and E C G Sudarshan have been of great help. Thanks are due to the organisers of ICOBAN, January 11-14, 1981, Bombay where the ideas were presented.

\section{References}

Coleman S and Weinberg S 1973 Phys. Rev. D7 1888

Dimopoulos S and Georgi 1981 Harvard Preprint HUTP-81/A022

Dimopoulos S, Raby S and Wilchek F 1981 Preprint NSF-ITP-S1-31

Dine M, Fischler W and Srednicki 1981 Inst. Adv. Study Preprint

Ferrara S and Piquet O 1975 Nucl. Phys. B93 261

Fischler W, Niles H P, Polochinski J, Raby S and Susskind L 1981 SLAC preprint

Gildner E 1976 Phys. Rev. D14 1667

Gildner E 1980 Phys. Lett. B92 111 
Gildner E and Weinberg S 1976 Phys. Rev. D13 333

Illiopoulos J and Zumino B 1974 Nucl. Phys. B76 310

Kaul R K 1981a CTS-TIFR Preprint TIFR/TH/81-32, (Phys. Lett. B in press)

Kaul R K 1981b Technicolour, CTS Preprint (revised)

Kaul R K and Majumdar P 1981a CTS Preprint

Kaul R K and Majumdar P 1981b CTS-TIFR Preprint, TIFR/TH/81-34 (Nucl. Phys. B in press)

Sakai N 1981 Tokoku University Preprints TU/81/225 and 226

Susskind L 1979 Phys. Rev. D20 2619

't Hooft G 1979 Lectures at Cargese Summer Institute

Veltman M 1980 Michigan Preprint

Wess J and Zumino B 1974a Nucl. Phys. B70 39

Wess J and Zumino B 1974b Phys. Lett. B49 52

Wess J and Zumino B 1974c Nucl. Phys.

Witten E 1981 Nucl. Phys. B188 513 